\title{
The influence of different growth conditions on the charge density wave transition of $1 T-\mathrm{TiSe}_{2}$
}

\author{
Matthias M. May*, Christine Brabetz, Christoph Janowitz, Recardo Manzke \\ Department of Physics, Humboldt-University of Berlin, Berlin, Germany
}

\begin{abstract}
To get insight into the influence of different growth conditions of $1 T-\mathrm{TiSe}_{2}$ onto its charge density wave (CDW) phase transition, we have performed angle-resolved photoelectron spectroscopy (ARPES) on $\mathrm{TiSe}_{2}$ in its CDW phase at low temperatures comparing the results with resistivity measurements. Though the local resistivity maxima become less pronounced with increasing growth temperatures, we do not find this trend in ARPES. While shape and intensity of the emission near $E_{F}$ change drastically, the backfolded valence bands are not affected. The question of the significance of the peaking resistivity as a characteristical 'fingerprint' of the CDW phase transition arises.
\end{abstract}

Keywords: Photoelectron spectroscopy, Charge density wave, $\mathrm{TiSe}_{2}$, Phase transition

\section{Introduction}

Charge density waves (CDWs) are a second-order phase transition into a state with a periodically modulated density of the charge carriers in solids. They have been a subject of interest for several decades being a collective charge carrier phenomenon often closely related to superconductivity. While the transition metal dichalcogenides and especially $\mathrm{TiSe}_{2}$ have been among the first materials where CDWs were discovered more than three decades ago [1], recent experiments $[2,3]$ revealed a competition between the CDW phase and a superconducting phase induced by $\mathrm{Cu}$ intercalation or pressure. A peaking resistivity in the CDW phase at a certain temperature is regarded as a 'fingerprint' of the transition with a strong dependence on the crystal growth conditions [1]. In this paper, we present ARPES and

\footnotetext{
* Corresponding author.

Email address: Matthias.May@physik.hu-berlin.de (Matthias M. May)
}

resistivity experiments investigating the influence of different growth conditions on the CDW phase transition of $\mathrm{TiSe}_{2}$.

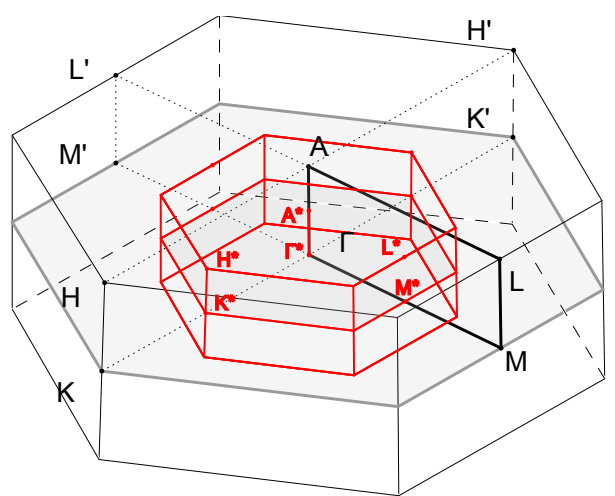

Figure 1: First Brillouin zone of $1 T-\mathrm{TiSe}_{2}$, in red the BZ of the $2 \times 2 \times 2$ superstructure in the CDW phase. 


\section{Experiment}

The $\mathrm{TiSe}_{2}$ single crystals (see fig. 1 for their BZ) were grown by iodine vapour transport using a four zone furnace with growth temperatures $\mathrm{T}_{g}$ of 740 and $630^{\circ} \mathrm{C}$. Their band structures were determined using synchrotron radiation $(h \nu=22 \mathrm{eV})$ at the beamline for education and scientific training (BEST) at the synchrotron source BESSSY II with a Gammadata Scienta 2002 analyser. An energy resolution of $25 \mathrm{meV}$ and an angle resolution of $0.1^{\circ}$ have been applied. With a liquid He cooled manipulator cryostat, the sample temperature was varied between $20 \mathrm{~K}$ and room temperature in UHV conditions of $<10^{-10}$ mbar and a temperature stability of $<1 \mathrm{~K}$. The samples were cleaved in situ to obtain clean surfaces. Resistivity measurements were performed with the four point contact method and a temperature alteration rate of $1 \mathrm{~K}$ per minute. The presented results compare resistivity respectively photoemission (ARPES) of one specific batch of samples for the two growth temperatures. Within one batch, they all showed the same trend and for $\mathrm{T}_{g}=740{ }^{\circ} \mathrm{C}$ this was confirmed for several separate batches.

\section{Results and discussion}

According to [1], $\mathrm{TiSe}_{2}$ shows an increasing nonstoichiometry, e.g. $\mathrm{Ti}_{1+x} \mathrm{Se}_{2}$, with higher growth temperatures, exhibiting $\mathrm{x}=0.01$ at $\mathrm{T}_{g}=800^{\circ} \mathrm{C}$ and $\mathrm{x}=0.018$ at $\mathrm{T}_{g}=900^{\circ} \mathrm{C}$. Their transport measurements reveal a strong dependence of the anomaly in resistivity in the plane perpendicular to the $\mathrm{c}$ axis corresponding to the growth temperature: Higher growth temperatures lead to a less pronounced peak in the temperature dependent resistivity curve as well as a shift of its maximum value towards lower temperatures. We can confirm this behaviour for our samples, crystals grown at $630{ }^{\circ} \mathrm{C}$ show a pronounced maximum in resistivity around $165 \mathrm{~K}$ while a growth temperature of $740{ }^{\circ} \mathrm{C}$ leads to a much less pronounced maximum at $155 \mathrm{~K}$, see fig. $2 \mathrm{~b}$ ). The curves exhibit no hysteresis. As an indicator for their sample purity, Di Salvo et al.[1] suggest ratio $\rho_{\max } / \rho(300 \mathrm{~K})$ values of up to 3 as well as Rossnagel et al. [4]. While we find $\rho_{\max }(165 \mathrm{~K}) / \rho(300 \mathrm{~K})=1.46$
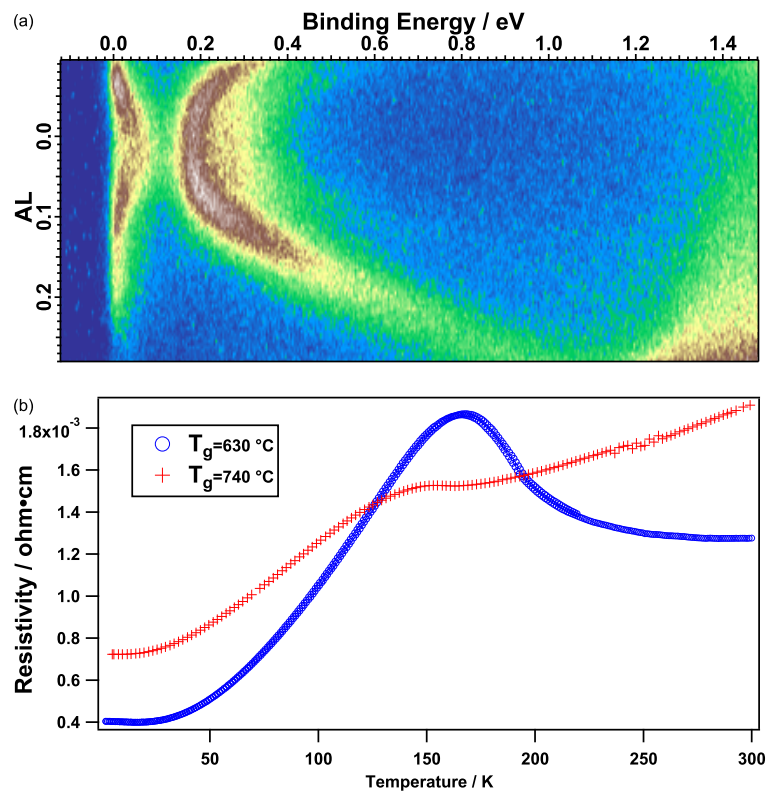

Figure 2: a) False colour photoemission intensity map at the $\mathrm{BZ}$ boundary in $\mathrm{AL}$ direction of a sample grown at $630^{\circ} \mathrm{C}$, measured at $22 \mathrm{~K}$. b) Resistivity $\perp$ to c direction.

and $\rho_{\max }(155 \mathrm{~K}) / \rho(300 \mathrm{~K})=0.8$ we doubt the significance of this parameter for the CDW phase transition due to our ARPES results.

The most distinct effect of the CDW phase transition visible in ARPES spectra is the backfolded, spin-orbit split Se 4p derived band appearing at the Brillouin zone (BZ) boundary, e.g. around the L point, see fig. 2a). Due to the $2 \times 2 \times 2$ superstructure, the $\mathrm{BZ}$ becomes renormalized and the valence band maximum of a new $\Gamma(A)$ point appears at $\mathrm{L}(\mathrm{M})$ of the unchanged BZ, see fig. 1. Therefore the intensity of the backfolded Se $4 p$ derived band at the BZ boundary can be viewed as the strength or order parameter of the CDW phase transition. Fig. 3a) shows an intensity contour plot of a sample grown at $740^{\circ} \mathrm{C}$, measured at the Brillouin zone (BZ) boundary $\mathrm{L}(\mathrm{M})$ in $\mathrm{A}(\Gamma) \mathrm{L}(\mathrm{M})$ direction at low temperature. One clearly identifies the backfolded valence band of Se $4 \mathrm{p}_{3 / 2}$ type around a binding energy $E_{B} \approx 180 \mathrm{meV}$ and at $E_{F}$ the Ti $3 d$ conduction band. For a detailed 

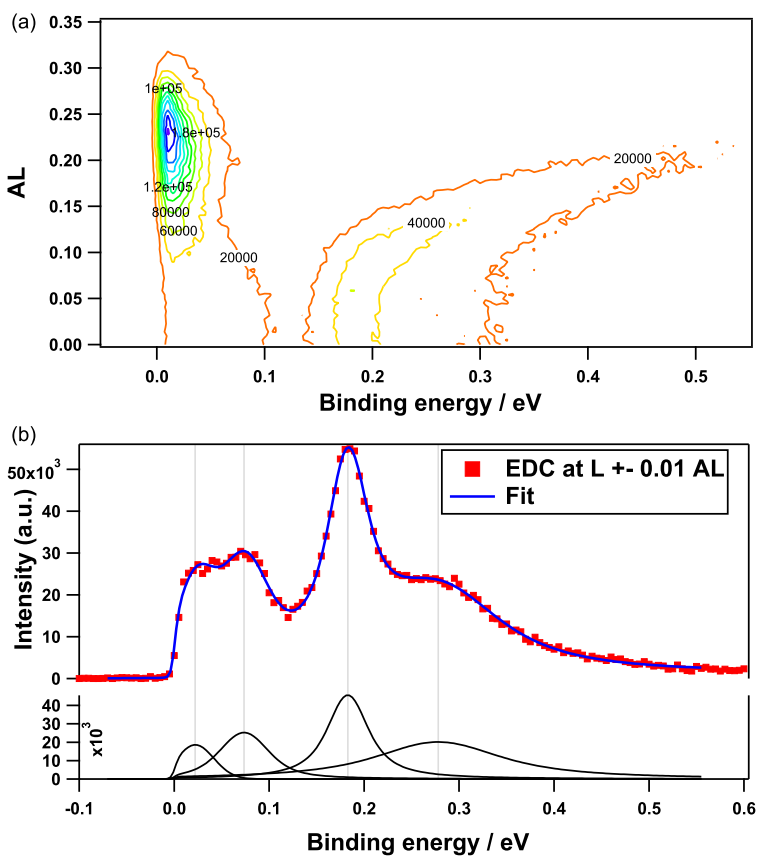

Figure 3: a) Intensity contour plot at the BZ boundary in AL direction of a sample grown at $740^{\circ} \mathrm{C}$, measured at $24 \mathrm{~K}$. b) EDC extracted at $\mathrm{L}(\mathrm{M})$, black curves show the contributions of the individual peaks to the overall fit.

analysis of their temperature evolution see e.g. [5]. Fig. 3b) shows an energy distribution curve (EDC) extracted exactly at the BZ boundary with fits to obtain the position of the peaks. We see two peaks located at 22 and $74 \mathrm{meV}$ and another two peaks at higher binding energies of 185 and $280 \mathrm{meV}$. The latter ones shift to lower binding energies with an increasing temperature. Notable here is the form of the Ti 3d emission at the Fermi energy evidencing a very sharp and pronounced peak located at $0.23 \mathrm{AL}$ with an intensity five times that of the backfolded bands at their maximum intensity. This could possibly be related to the band $c_{3}$ mentioned in [6]. An attempt to quantify the evolution of this artefact could be the fraction of the artefact's maximum intensity to the intensity of the band near $E_{F}$ exactly at the BZ boundary. In the case presented here, this is about 7.4 for $\mathrm{T}_{g}=740{ }^{\circ} \mathrm{C}$ and 1.6 for $\mathrm{T}_{g}=630^{\circ} \mathrm{C}$.

This artefact of the conduction band is obviously related to nonstoichiometric $\mathrm{Ti}$ atoms, probably due to their intercalation into the van der Waals gap and increasing occupation of the conduction band. The results for a sample grown at $630^{\circ} \mathrm{C}$ are shown in fig. 4. Here, we do not find such an intense peak in the Ti $3 \mathrm{~d}$ emission, the maximum intensity of the bands near $E_{F}$ is slightly smaller than the intensity of the peaks found in the backfolded band. Still, the backfolded bands can be clearly identified and the positions of the four peaks at 11, 60, 180 and $270 \mathrm{meV}$ are very similar to the locations identified for the crystal grown at $740{ }^{\circ} \mathrm{C}$ discussed above. Although the indications of the strength of the CDW transitions in both crystals are the same, it seems to be that the backfolded Se $4 \mathrm{p}$ bands can be traced to slightly higher binding energies for the crystal grown at $630^{\circ} \mathrm{C}$.
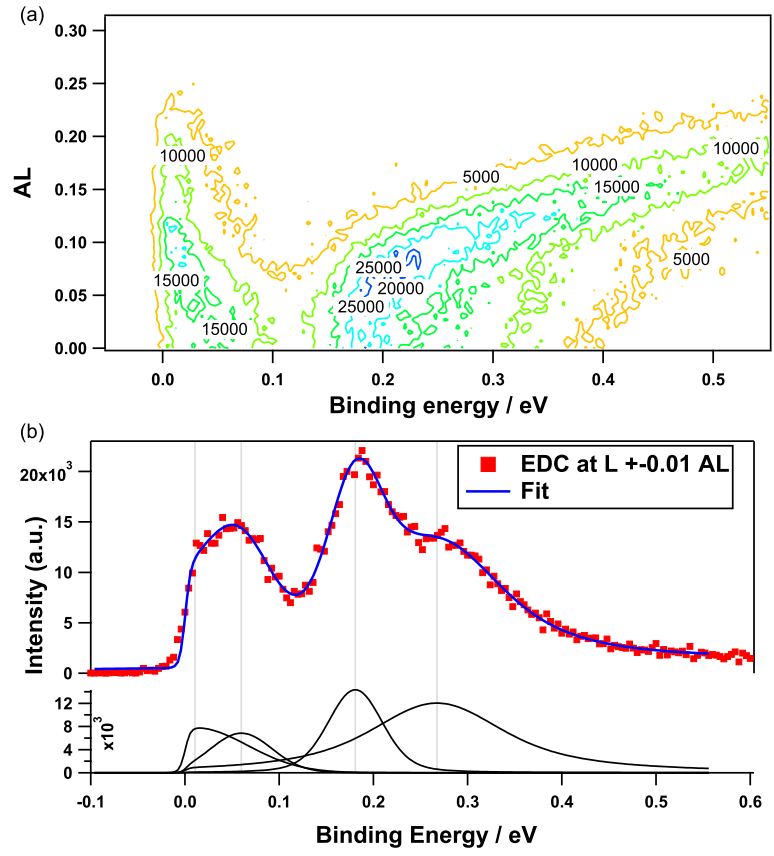

Figure 4: a) Intensity contour plot at the BZ boundary in $\mathrm{AL}$ direction of a sample grown at $630{ }^{\circ} \mathrm{C}$, measured at $22 \mathrm{~K}$. b) EDC extracted at $\mathrm{L}(\mathrm{M})$, black curves show the contributions of the individual peaks to the overall fit.

The similar CDW strength observed in photoe- 
mission is in clear contrast with the transport data where we observe a dramatic decrease of the resistivity anomaly with increasing growth temperature. Therefore our observations do not confirm the longstanding assumption since the work of Di Salvo et al. [1] that a pronounced resistivity anomaly is indicative for the strength of the charge density wave state in $\mathrm{TiSe}_{2}$. The microscopic effect of backfolding should be viewed as much more significant for the CDW phase transition than the anomaly in resistivity. This is in line with the fact that the backfolded bands remain observable up to temperatures distinctly higher than the so-called transition temperature determined from the resistivity data. Monney et al. [6] found the umklapp bands, although quite weak, at least up to room temperature, similar to our observations.

In the excitonic condensate phase model for the driving force of the CDW transition, Monney et al. [6] quantify the strength of the CDW phase with an order parameter $\Delta$, which is proportional to the energy gap between the backfolded bands and the Ti $3 \mathrm{~d}$ emission. They found very similar positions of the four contributions of the signal [5]. Our results regarding the positions of the bands seem to support their view. However, we find that for the higher growth temperature the backfolded valence band has a higher relative intensity. This suggests that an indicator for the strength of the CDW phase transition derived from relative intensities of backfolded bands to the bands near $E_{F}$ could be more adequate. In our case, this would be approximately 1.8 for the higher growth temperature and 1.4 for the lower temperature (extracted from the EDCs in fig. 3 and 4 ).

\section{Conclusion}

The influence of different growth temperatures between $T_{g}=630^{\circ} \mathrm{C}$ and $740^{\circ} \mathrm{C}$ on the $\mathrm{CDW}$ phase transition of $1 T-\mathrm{TiSe}_{2}$ with respect to resistivity and ARPES data has been investigated. Higher growth temperatures lead to a less pronounced peaking or anomaly in the temperature dependent resistivity and a transfer of more spectral weight to the Ti $3 \mathrm{~d}$ bands as well as a change of their shape. However, the appearance of the backfolded valence bands is not affected. This questions the relevance of the resistivity anomaly for the characterisation of the CDW phase transition. On the other hand, the transition is excellently characterised in a microscopic manner by the appearance of backfolded bands in the Brillouin zone and we suggest to take the intensity of these bands to quantify the order of the phase transition.

\section{Acknowledgements}

Thanks to Dr. H. Dwelk for his help with the resistivity measurements and to Stephan Thürmer for the helpful discussions. Part of the work has been conducted at BESSY. We thank the staff and in particular Dr. Gerd Reichardt.

\section{References}

[1] F. J. Di Salvo, D. E. Moncton, J. V. Waszczak, Phys. Rev. B 14 (1976) 4321.

[2] E. Morosan, H. Zandbergen, B. Dennis, J. Bos, Y. Onose, T. Klimczuk, A. Ramirez, N. Ong, R. Cava, Nat. Phys. 2 (2006) 544.

[3] A. Kusmartseva, B. Sipos, H. Berger, L. Forro, E. Tutis, Phys. Rev. Lett. 103 (2009) 236401.

[4] K. Rossnagel, L. Kipp, M. Skibowski, Phys. Rev. B 65 (2002) 235101.

[5] C. Monney, E. F. Schwier, M. G. Garnier, N. Mariotti, C. Didiot, H. Beck, P. Aebi, H. Cercellier, J. Marcus, C. Battaglia, H. Berger, A. N. Titov, Phys. Rev. B 81 (2010) 155104.

[6] C. Monney, H. Cercellier, F. Clerc, C. Battaglia, E. F. Schwier, C. Didiot, M. G. Garnier, H. Beck, P. Aebi, H. Berger, L. Forró, L. Patthey, Phys. Rev. B 79 (2009) 045116. 